\title{
Self-Assembling Multidomain Peptide Nanofibers for Delivery of Bioactive Molecules and Tissue Regeneration
}

Published as part of the Accounts of Chemical Research special issue "Stimuli-Responsive Hydrogels".

Amanda N. Moore ${ }^{\dagger}$ and Jeffrey D. Hartgerink ${ }^{*},+\neq 0$

${ }^{\dagger}$ Department of Chemistry, Rice University, 6500 Main Street, Houston, Texas 77005, United States

${ }^{\ddagger}$ Department of Bioengineering, Rice University, 6500 Main Street, Houston, Texas 77005, United States

Supporting Information

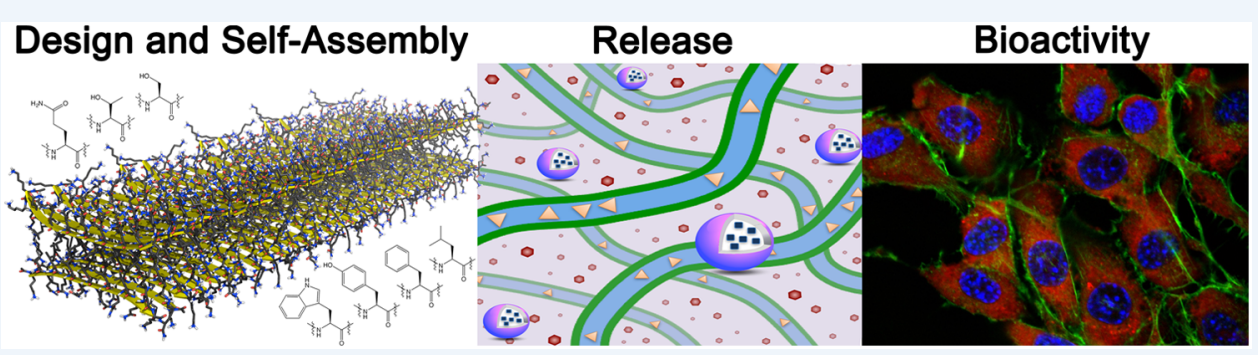

CONSPECTUS: Multidomain peptides (MDPs) are a class of self-assembling peptides that are organized in a $\beta$-sheet motif, resulting in a nanofibrous architecture. This structure is stabilized by hydrophobic packing in the fiber core and a hydrogenbonding network down the fiber long axis. Under easily controllable conditions, regulated by electrostatic interactions between the peptides and the $\mathrm{pH}$ and salt composition of the solvent, the nanofiber length can be dramatically extended, resulting in fiber entanglement and hydrogel formation. One of the chief strengths of this supramolecular material is that the design criteria governing its structure and assembly are robust and permit a wide range of modifications without disruption. This allows the MDPs to be tailored to suit a wide range of applications, particularly in biomedical engineering. For example, delivery of small molecules, proteins, and cells is easily achievable. These materials can be trapped within the matrices of the hydrogel or trapped within the hydrophobic core of the nanofiber, depending on the cargo and the design of the MDP. Interactions between the nanofibers and their cargo can be tailored to alter the release profile, and in the most sophisticated cases, different cargos can be released in a cascading time-dependent fashion. The MDP hydrogel and its cargo can be targeted to specific locations, as the thixotropic nature of the hydrogel allows it to be easily aspirated into a syringe and then delivered from a narrow-bore needle. The sequence of amino acids making up the MDP can also be modified to permit cross-linking or enzymatic degradation. Selection of sequences with or without these modifications allows one to control the rate of degradation in vivo from as rapidly as 1 week to well over 6 weeks as the MDP nanofibers are degraded to their amino acid components. MDP sequences can also be modified to add biomimetic sequences derived from growth factors and other signaling proteins. These chemical signals are displayed at a very high density on the fibers' surface, where they contribute to the modification of cellular behavior. We have used this approach to drive blood vessel formation, which is critical for tissue regeneration generally and more specifically for the treatment of diseases related to poor blood flow. MDPs represent an ideal case of bottom-up design where control of chemical structure leads to control of self-assembly and nanostructure and thereby control of material properties that collectively can control biological function.

\section{INTRODUCTION}

While primarily composed of water, hydrogels can be prepared from both natural and synthetic materials, including both small molecules and polymers. Perhaps the most complex hydrogel of all is the extracellular matrix (ECM), which plays a critical role in determining tissue architecture and cellular behavior. Because the ECM has a large role in cell adhesion, cell survival, cell proliferation, cell signaling, cell differentiation, and nutrient/waste exchange, many attempts have been made to mimic its structure, materials properties, and ultimately its function. Among these attempts, peptide hydrogels ${ }^{1}$ and peptide-derivatized hydrogels ${ }^{2}$ have been particularly successful.
While peptide hydrogels can utilize various structural motifs to drive supramolecular assembly and hydrogel formation, perhaps the most explored category for tissue regeneration purposes is the $\beta$-sheet peptide hydrogel. Several $\beta$-sheet hydrogels have been developed, synthesized, and characterized for biomedical applications, including multidomain peptides (MDPs) developed by our lab. Other key examples include peptide amphiphiles from the Stupp lab, ${ }^{3}$ RADA-16 and derivatives from the Zhang lab, ${ }^{4}$ and $\beta$-hairpin peptides from the Schneider

Received: November 3, 2016

Published: February 13, 2017 
and Pochan labs. ${ }^{5}$ This Account does not discuss all of the $\beta$ sheet peptide hydrogels proposed in the literature but rather presents the development of self-assembling MDP hydrogels by our lab. We recommend several reviews that present broader perspectives on peptide-based and other hydrogels. ${ }^{1,6-10}$

For the past decade, our lab has worked to engineer a class of self-assembling peptide hydrogels that we call multidomain peptides. With solely the 20 naturally occurring amino acids as building blocks, these peptides are synthesized using standard solid-phase chemistry and require no special functionality for supramolecular assembly or hydrogelation. By relying on principles of supramolecular chemistry, we have designed this class of $\beta$-sheet peptides to self-assemble into nanofibers using the composition of the aqueous solution as the trigger for assembly. The design of the peptide primary sequence enables cross-linking of peptide nanofibers either ionically or covalently to induce hydrogelation. The MDP hydrogel has demonstrated great promise as a bioengineering scaffold, as the triggering conditions for peptide self-assembly and cross-linking are compatible with physiological conditions. We have demonstrated that the MDP hydrogel is biocompatible with multiple cell types, can be used to effectively deliver cells or various types of bioactive molecules, and can be altered to include bioactive amino acid sequences that direct cell behavior. Through simple changes in the chemical sequence of the peptide, we can dictate the formation of nanofibers through self-assembly, the formation of a hydrogel through entanglement of the formed fibers, and the mechanical properties of the formed hydrogel. Ultimately, this allows us to also dictate the in vivo response to the MDP hydrogel through a combination of these properties and associated biological signals encapsulated within the hydrogel or incorporated into the assembling peptide. In principle, therefore, engineering of the MDP primary sequence allows for manipulation of the scaffold to suit any specific biological application. While other related peptide hydrogels have some of these features, the combination of all of them make MDPs uniquely suited to biological applications.

\section{PART 1: PEPTIDE DESIGN}

Initial studies performed by our laboratory revealed how the peptide sequence can be designed to allow for the supramolecular assembly of individual peptides to form fibers. All MDPs exhibit the same general chemical design, with the core of the peptide consisting of alternating hydrophilic and hydrophobic amino acids and the termini of the peptide consisting of charged amino acids (Figure 1A,B). ${ }^{11}$ As shown in Figure $1 \mathrm{~A}$ and SI Table 1, we have investigated a variety of different amino acids to compose each motif within the MDP. By using glutamate or lysine as the charged terminal residues, we have generated peptides with net negative or positive charge, respectively. ${ }^{12}$ Hydrophilic residues have included glutamine, serine, threonine, and cysteine, while hydrophobic residues have included both aliphatic and aromatic side chains. ${ }^{1-14}$ Regardless of the specific amino acids selected to compose the peptide, the chemical design of the MDP causes these peptides to adopt a $\beta$-sheet secondary structure with hydrophobic residues on one face of the peptide and hydrophilic residues on the other. Arising from the alternating hydrophilic and hydrophobic residues in the core of the MDP, this facial amphiphile is key for the supramolecular assembly of peptides to form nanofibers.

Because of the amphiphilic nature of MDPs, they selfassemble to form nanofibers in aqueous solution. Each fiber
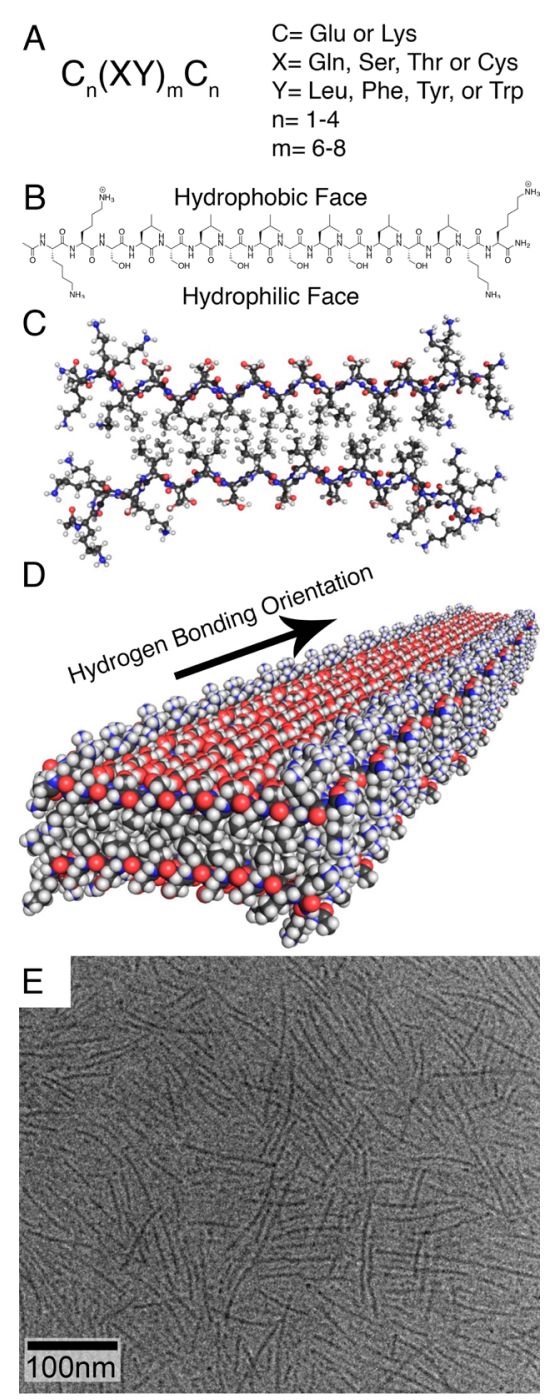

Figure 1. Self-Assembly of MDPs. (A) General composition of MDPs. (B) Primary sequence of an example MDP, $\mathrm{K}_{2}(\mathrm{SL})_{6} \mathrm{~K}_{2}$. (C) Repeating unit of the MDP nanofiber. (D) Depiction of an MDP nanofiber. (E) Vitreous ice cryo-TEM image of the self-assembled MDP $\mathrm{K}_{2}(\mathrm{QL})_{6} \mathrm{~K}_{2}$ before the addition of counterions. (C, D) Adapted with permission from ref 15. Copyright 2014 Elsevier. (E) Reprinted from ref 11. Copyright 2007 American Chemical Society.

consists of a bilayer of peptides with the hydrophilic peptide faces exposed to the surrounding aqueous solution and the hydrophobic peptide faces sequestered into the core of the MDP. Intellectually, it is convenient to divide the fibers into repeating units to understand why MDPs undergo supramolecular assembly. We call the repeating unit of the MDP nanofiber a "hydrophobic sandwich", and it comprises four peptides (Figure 1C). ${ }^{15}$ In this sandwich, the hydrophobic faces of the MDPs pack against one another to minimize contact with surrounding water molecules. When aligned, hydrogen bonding between adjacent peptide backbones can occur, further stabilizing the assembled peptides (Figure 1D). Our lab has also discovered methods of defining the peptide orientation within the nanofibers, specifically the formation of parallel or antiparallel hydrogen-bonding networks. For instance, most MDPs contain leucine residues as their hydrophobic core, and all of these sequences exhibit antiparallel packing of the $\beta$-sheet peptides. ${ }^{11-14,16,17}$ However, by the use of tryptophan or tyrosine in place of leucine, it is possible to change this 


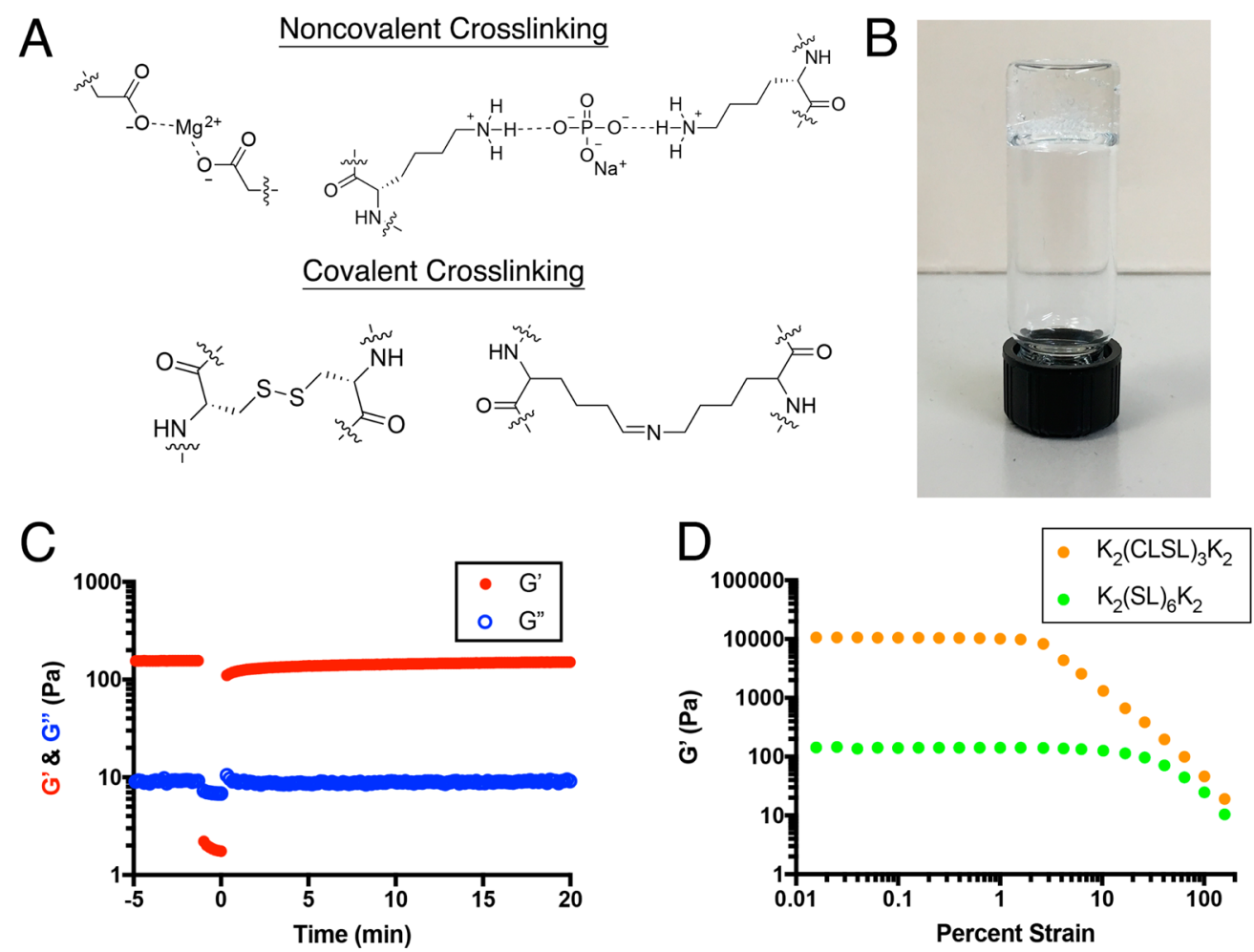

Figure 2. (A) Various methods to cross-link MDPs using non-covalent or covalent interactions. (B) Cross-linking results in a clear hydrogel. (C) Shear recovery of the MDP hydrogel. At time $=-1$, a shearing force is applied to liquefy the hydrogel, and at time $=0$ the shearing force is released. (D) Strain sweep analysis of a covalently cross-linked MDP hydrogel vs a non-covalently cross-linked MDP hydrogel. Adapted from refs 12 and 29. Copyright 2009 and 2016 American Chemical Society.

orientation to parallel packing of the peptides. Modeling suggests that this is the case because the side chains of these two amino acids form stronger interactions in the hydrophobic sandwich when fibers self-assemble in a parallel rather than antiparallel fashion, thus defining the hydrogen-bonding orientation. ${ }^{14}$ While not mechanistic in nature, the idea of the hydrophobic sandwich illustrates the concepts driving selfassembly of MDPs. Theoretical studies by other groups have also been performed in attempts to elucidate the actual step-bystep process of peptide assembly. ${ }^{18}$ Regardless of the mechanism of supramolecular assembly, the final result is a nanofiber, exhibiting the width of the peptide sequence, the height of the MDP dimer, and "infinite" length after charge screening (Figure 1E). ${ }^{11}$ To characterize MDPs, we confirm successful synthesis using mass spectrometry; the secondary structure using circular dichroism spectroscopy and infrared spectroscopy; self-assembly using atomic force microscopy, scanning electron microscopy, and/or transmission electron microscopy; and hydrogel formation using oscillatory rheology.

With the goal of creating self-assembled nanofibrous structures while maintaining solubility, the proper balance of charged residues to hydrophilic-hydrophobic residue repeats was determined experimentally. Through circular dichroism (CD) spectroscopy and transmission electron microscopy (TEM), it was found that multidomain peptides typically require at least 3 times more hydrophilic-hydrophobic repeats than charged residues to form nanofibers. ${ }^{11}$ When too few charged residues are included in the design, the peptides have very poor solubility and are difficult to handle and purify. However, if the peptide contains too many charged residues, fibers fail to form as a result of like-charge repulsion at the peptide termini. In terms of hydrophilic-hydrophobic amino acid repeats, a peptide without a sufficient number will either fail to form fibers or form fibers contaminated with amorphous aggregates of assembled peptides. By properly balancing the number of charged residues with the number of hydrophilichydrophobic residue repeats, it is possible to consistently obtain self-assembled nanofibers of relatively uniform length and diameter (Figure 1E).

MDPs are further engineered at the primary sequence level to allow for simple and biologically compatible hydrogelation through cross-linking of peptide nanofibers (Figure 2A,B). The inclusion of charged residues at the peptide termini serves two purposes: first, to increase the solubility, and second, to enable non-covalent cross-linking between peptide fibers. In aqueous solution, the charged residues at the peptide termini oppose fiber self-assembly; however, the addition of multivalent oppositely charged salts triggers hydrogelation by using these charged residues as locations to cross-link fibers. For positively charged peptides, we typically use a phosphate buffer for crosslinking, while a magnesium or calcium salt is appropriate for cross-linking of negatively charged peptides. Monovalent salts such as $\mathrm{NaCl}$ also work but require much higher concentrations. If desired for a specific application, it is also possible to cross-link peptides using drug molecules of the appropriate charge. ${ }^{19}$ Significantly, these non-covalent interactions are capable of reformation after disruption, allowing the MDP hydrogel to be a thixotropic material that liquefies under a shearing force and regels when the shearing force ceases. We have demonstrated this using oscillatory rheology. When a shearing force is applied, the storage and loss moduli invert, signifying liquification. Following removal of the shearing force, the storage and loss moduli recover to approximately $75 \%$ of their initial values nearly immediately and to $100 \%$ over $15 \mathrm{~min}$ 

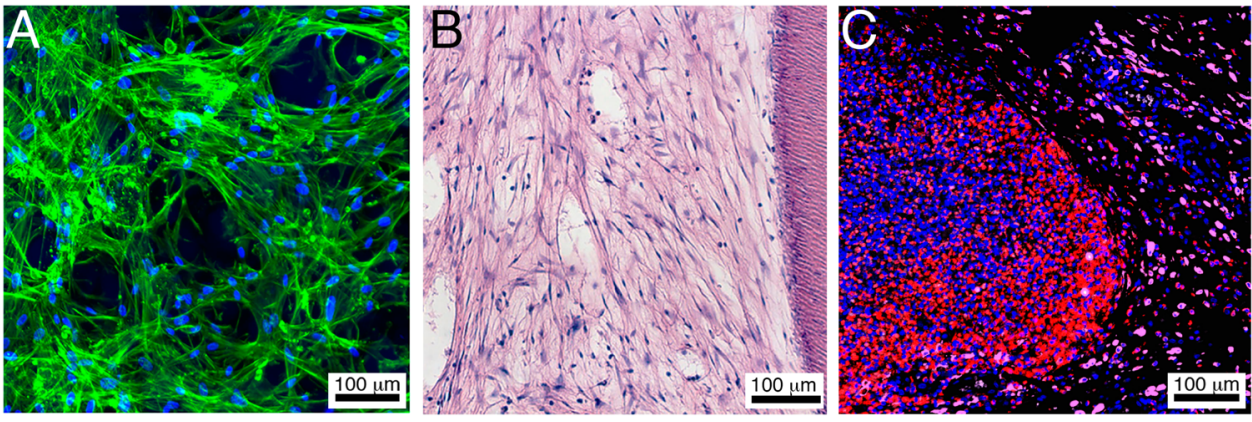

Figure 3. (A) Confocal images of stem cells from human deciduous teeth (SHED) cells after 11 days of $3 \mathrm{D}$ culture in $\mathrm{K}_{2}(\mathrm{SL})_{6} \mathrm{~K}_{2}$ hydrogel. Cell nuclei are stained with DAPI and appear blue, while the actin cytoskeleton is stained with AlexaFluor 488 and appears green. Reprinted from ref 13 . Copyright 2014 American Chemical Society. (B) H\&E image of pulplike tissue formed with concurrent delivery of dental pulp stem cells (DPSCs) and the growth factors VEGF, TGF $\beta 1$, and FGF2. Reprinted with permission from ref 25. Copyright 2012 Mary Ann Liebert, Inc. (C) Immunohistological analysis of a subcutaneous $\mathrm{K}(\mathrm{SL})_{3} \mathrm{RG}(\mathrm{SL})_{3} \mathrm{KGRGDS}$ hydrogel excised 1 week after implantation. Cell nuclei appear blue, $\mathrm{CCR}^{+}$appear green, $\mathrm{CD}_{163^{+}}$appear purple, and $\mathrm{CD} 68^{+}$appear red. Reprinted with permission from ref 23. Copyright 2015 Elsevier.

(Figure 2C). In practical terms, the ability to shear thin and shear recover is an extremely valuable property of the MDP hydrogel, as it enables the material to be easily delivered via injection. We have relied on this property for several of our biological studies, and we have successfully delivered the MDP hydrogel using as small as a 33 gauge needle. ${ }^{20}$

While our lab most commonly uses non-covalent crosslinking with salt solutions to trigger hydrogelation, we have extensively investigated other methods of cross-linking as well. With the motivation of manipulating the rheological properties of the MDP hydrogel, we have studied covalent cross-linking of peptide nanofibers. Because covalent cross-linking forms stronger interactions between peptide fibers, a dramatic increase in storage modulus is observed for covalently crosslinked MDP hydrogels. ${ }^{12,21}$ One method of covalent crosslinking uses cysteine residues as hydrophilic amino acids in the core of the MDP. For this strategy, general oxidation results in intrafiber and interfiber covalent disulfide bonds. The disulfide bonds formed between two cysteine residues result in a dramatic increase in gel storage modulus (Figure 2D). ${ }^{12}$ It is also possible to utilize enzymes to covalently cross-link peptide fibers. For instance, lysyl oxidase is an enzyme found in nature that functions to cross-link ECM components. In nature, it acts by oxidizing an amine to an aldehyde, which can then react with another amine to form a Schiff base or undergo an aldol condensation reaction with another aldehyde. In our system, lysyl oxidase acts on the lysine side chains of the MDP and covalently cross-links peptide nanofibers. This increases the storage modulus by over an order of magnitude in comparison to the non-covalently cross-linked system of the same MDP. ${ }^{21}$

While MDPs are relatively short in length, typically between 18 and 30 amino acids, careful selection of these amino acids results in the desired chemical and mechanical properties. Alternation of hydrophilic and hydrophobic residues drives $\beta$ sheet formation in either a parallel or antiparallel orientation. Charged residues at the peptide termini determine the overall scaffold charge and therefore which molecules can be used to non-covalently cross-link the peptide nanofibers. To influence the mechanical properties, non-covalent or covalent crosslinking can be used. Through the design of the peptide primary sequence, we have created a self-assembling nanofibrous scaffold capable of delivery via syringe injection.

\section{PART 2: LOADED HYDROGELS}

The fibrous nanostructure of the MDP enables these hydrogels to be effective vehicles for culture and/or delivery of cells and other bioactive molecules. During formation of the hydrogel, these substances can easily be included, resulting in a $3 \mathrm{D}$ injectable hydrogel containing the substance of interest. Because of differences in relative size, cells tend to remain trapped within the MDP fibrous matrix, while molecules typically diffuse out of the hydrogel. Because of this, the MDP hydrogel can serve as an effective material to evaluate cell behavior in a 3D environment, and this constitutes an important advantage because there is wide consensus in the literature that cell behavior differs drastically in $2 \mathrm{D}$ and $3 \mathrm{D}$ culture methods. ${ }^{22}$ We have completed several studies with a variety of cell types, such as stem cells and monocytes, to confirm the biocompatibility of the MDP hydrogel and evaluate cell behavior in $2 \mathrm{D}$ versus $3 \mathrm{D}$ culture (Figure $3 \mathrm{~A}$ and SI Table 2). For many cell types, the MDP hydrogel suggests a high level of biocompatibility, showing cell adhesion to the scaffold, cell proliferation, and adoption of characteristic cell morphology.

In addition to cell culture and delivery, we have also evaluated the potential for the MDP hydrogel to effectively carry and deliver hydrophilic bioactive molecules. We have previously published MDP release data for a wide variety of bioactive molecules, including $\mathrm{IL}-4,{ }^{23} \mathrm{MCP}-1,{ }^{23,24} \mathrm{VEGF}{ }^{25}$ TGF $\beta 1,{ }^{25}$ FGF $2,{ }^{25}$ PlGF- $1,{ }^{24} \mathrm{EGF}^{24}$ and batroxobin, ${ }^{26}$ but in theory the MDP hydrogel could be used to deliver virtually any bioactive molecule. The motivations driving different release studies vary greatly; we have examined treatment of perioperative bleeding, kidney injury, and tooth decay (Figure 3B), modulation of inflammation (Figure 3C), and promotion of angiogenesis. ${ }^{17,23-28}$ While the biological applications of these studies differ significantly, the results consistently show that the MDP hydrogel can effectively load and release bioactive molecules and that these molecules remain functional and are able to provoke a biological response after delivery. For reasons not yet entirely understood, the release rates of molecules from the hydrogel differ depending on molecule identity. For instance, MCP-1 exhibits burst release from the MDP hydrogel, while release of IL-4 is sustained over a few weeks. ${ }^{23}$ We have speculated that hydrodynamic volume or molecular mass play a role in defining the release profile, but other factors such as specific binding interactions must also impact the release kinetics. 
Changes in MDP primary sequence can also be used to engineer the types of molecules that can be effectively loaded and delivered by the MDP hydrogel. Because the MDP design incorporates charged, hydrophilic, and hydrophobic amino acids, we are able to deliver molecules of varying polarity. In some ways this is counterintuitive, as a hydrogel is by definition aqueous and therefore should be more suited to deliver hydrophilic molecules. However, each MDP fiber contains a hydrophobic core, and hydrophobic molecules can be successfully encapsulated within MDP fibers. Thus, hydrophilic molecules are encapsulated external to the MDP fibers, while hydrophobic molecules are encapsulated within the actual MDP fiber itself (Figure 4A,B). To allow for encapsulation of

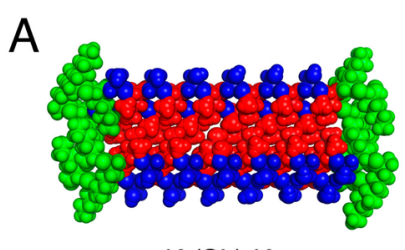

$\mathrm{K}_{2}(\mathrm{SL})_{6} \mathrm{~K}_{2}$

Repeating Unit

C

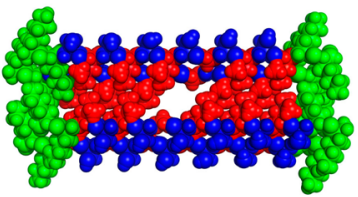

$\mathrm{K}_{2}(\mathrm{SL})_{2} \mathrm{SA}(\mathrm{SL})_{3} \mathrm{~K}_{2}$

Repeating Unit

E

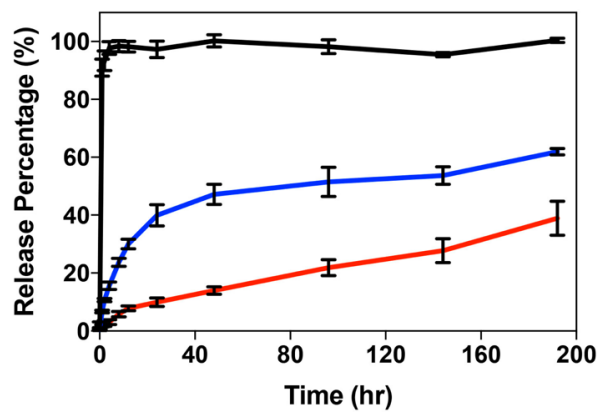

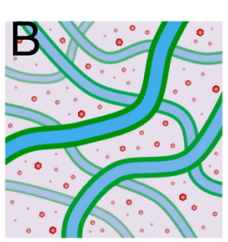

Fast drug release by diffusion

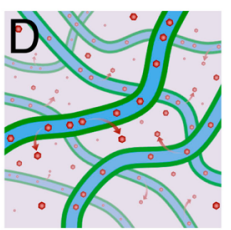

Prolonged drug release

by intrafibrillar encapsulation
Figure 4. (A, B) Typical repeating unit of an MDP that results in fast release of drugs. (C, D) Missing-tooth MDP repeating unit allowing for drug encapsulation in the nanofiber core and resulting in prolonged release. (E) Data showing rapid release of etodolac from PBS (black), slower release of etodolac from the $\mathrm{K}_{2}(\mathrm{SL})_{6} \mathrm{~K}_{2}$ hydrogel, and prolonged release from the missing-tooth $\mathrm{K}_{2}(\mathrm{SL})_{2} \mathrm{SA}(\mathrm{SL})_{3} \mathrm{~K}_{2}$ hydrogel. Adapted from ref 29. Copyright 2016 American Chemical Society.

hydrophobic molecules, we engineered a gap into the core of the MDP, and we refer to this design as the "missing-tooth" model (Figure 4C,D). ${ }^{29}$ Essentially, one or two of the leucine residues in the core of the peptide is altered to an alanine residue. Because alanine has a smaller side chain than leucine, this creates space along the core of the MDP fiber into which hydrophobic materials can be successfully loaded while not preventing fiber formation or gelation. For instance, the unmodified $\mathrm{K}_{2}(\mathrm{SL})_{6} \mathrm{~K}_{2}$ hydrogel has a storage modulus of $191 \mathrm{~Pa}$, and the storage moduli of the $\mathrm{K}_{2}(\mathrm{SL})_{2} \mathrm{SA}(\mathrm{SL})_{3} \mathrm{~K}_{2}$ and $\mathrm{K}_{2}(\mathrm{SL})_{2}(\mathrm{SA})_{2}(\mathrm{SL})_{2} \mathrm{~K}_{2}$ hydrogels are each roughly equivalent at 142 and 158 Pascals, respectively. ${ }^{29}$ We have demonstrated hydrophobic loading and delivery using SN-38, diflunisal, and etodolac, which suggests that this strategy should allow for the entrapment and delivery of virtually any appropriately sized molecule with poor water solubility (Figure 4E). ${ }^{29}$

In addition to defining the types of materials that can be delivered by the MDP hydrogel, it is also possible to alter the MDP hydrogel structure to engineer the release rate of encapsulated materials. To accomplish this, our lab has introduced an othogonal level of supramolecular assembly by incorporating liposomes into the MDP hydrogel (Figure 5A,B).

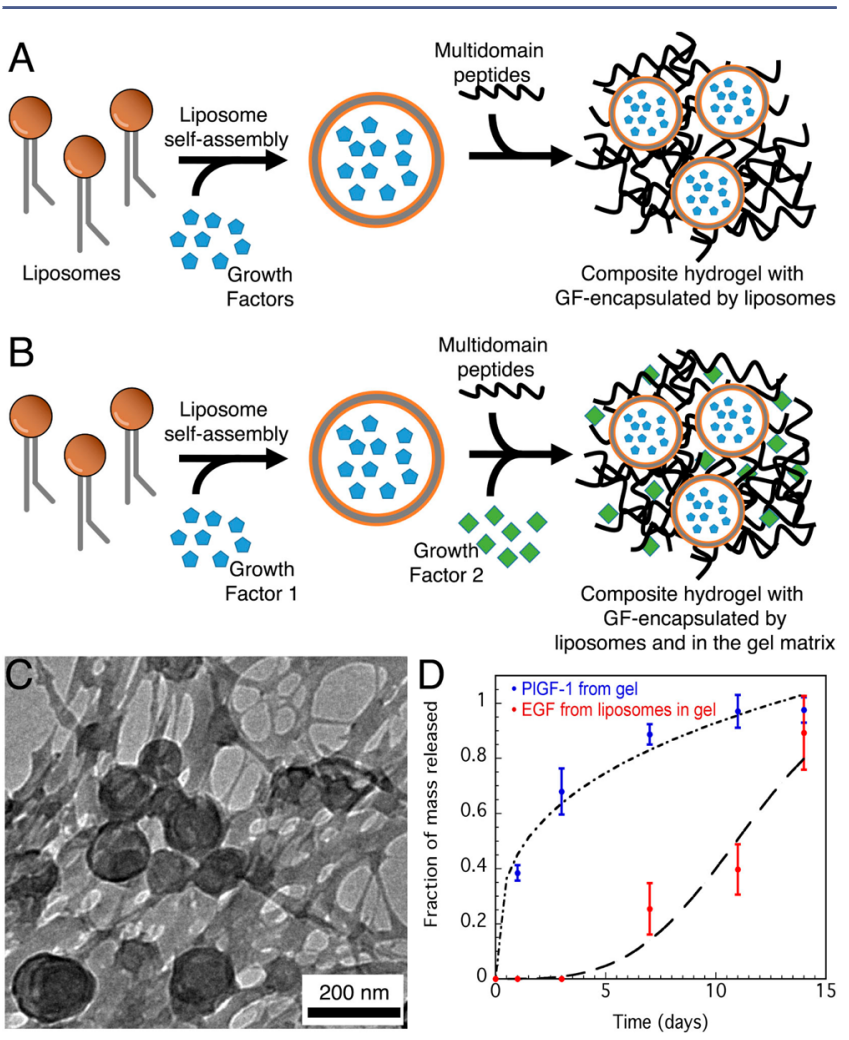

Figure 5. (A) Schematic showing self-assembly of peptide hydrogels with growth factors loaded inside of liposomes. (B) Schematic of selfassembly with one growth factor loaded inside of liposomes and another loaded into the MDP hydrogel. (C) Cryo-TEM image of the liposome-MDP composite hydrogel. (D) Release of PlGF-1 directly from the hydrogel (blue) and release of EGF from liposomes encapsulated within the hydrogel (red). Adapted from ref 24. Copyright 2014 American Chemical Society.

Liposomes and peptide nanofibers are distinct supramolecular assemblies, but they can be mixed together without interrupting the integrity of either structure (Figure 5C). ${ }^{24}$ Furthermore, this design engineers a system for biphasic release of molecules from the MDP hydrogel. Material loaded inside the MDP hydrogel but outside the liposomes is released more rapidly than material loaded inside the liposomes. This is the case because liposomal degradation takes time to occur and has to take place prior to diffusion of material from the MDP hydrogel. We have demonstrated the proof of concept by loading PlGF-1, MCP-1, and EGF either into liposomes or directly into the MDP; when loaded into liposomes, all three molecules show a sigmoidal release profile, while direct loading into the MDP hydrogel results in burst release (Figure 5D). ${ }^{24}$ Biologically speaking, the capability of biphasic release has important implications in the applicability of MDP hydrogels. Most biological processes involve signaling molecules released 
in a specific temporal order, and we have engineered a scaffold capable of delivering materials in a temporally distinct manner by creating these liposome-MDP composite hydrogels.

The design of the peptide primary structure ultimately results in a $3 \mathrm{D}$ material capable of effectively delivering and releasing bioactive molecules and supporting cell viability. Although our studies have involved a variety of different cell types, bioactive molecules, and applications, the supramolecular engineering of MDPs to form nanofibrous hydrogels is collectively responsible for effective loading and delivery. While the base MDP hydrogel is capable of delivering water-soluble materials, small alterations in the peptide primary structure allow for MDPbased delivery of hydrophobic molecules as well. Furthermore, temporal control over material delivery can be achieved through incorporating additional structures within the hydrogel matrix. Importantly, delivering materials using MDP hydrogels preserves the bioactivity of molecules and vitality of cells and offers a method for localized application through injection.

\section{PART 3: BIOACTIVITY AND BIOMIMICRY}

While MDP hydrogels can be designed to effectively deliver exogenous growth factors, cytokines, and drug molecules, these materials can also be synthesized to have distinct biofunctionality of their own through alteration of the peptide primary sequence. Researchers have identified short bioactive amino acid sequences, typically derived from biological molecules, that are capable of initiating specific responses from cells. Because of the modular nature of solid-phase peptide synthesis, these short sequences can easily be attached to the termini of the MDP and in some cases incorporated into the middle of the MDP sequence. Because of the robust and well-understood assembly of MDPs, the incorporation of these types of short signaling motifs can typically be accomplished without interrupting the characteristic and desirable properties of MDP hydrogels, such as their fibrous nanostructure and rheological properties. For example, our lab has investigated MDPs designed to promote cell adhesion, aid in material degradation, and drive angiogenesis.

In order to preserve vitality and other basic cellular functions, many cell types require adherence to their environment, the ECM. Because the MDP hydrogel mimics the native ECM in terms of its fibrous nanoscale structure, cells are capable of adhering to the MDP fibers; furthermore, this response by cells can be stimulated through the addition of a bioactive amino acid sequence specifically designed to aid in cell adhesion. Derived from the ECM protein fibronectin, RGD has been widely cited for its ability to increase cellular adhesion to biomaterial scaffolds both in vitro and in vivo. We have incorporated this sequence at the C-terminus of the base MDP and evaluated its impact on the responsiveness of cells to the matrix. ${ }^{13,17,20,25}$ In in vitro experiments, we have found that inclusion of RGD promotes cell viability, spreading, and proliferation, suggesting increased vitality of cells in the bioactive scaffold (Figure 6). ${ }^{13,25}$

Gel responsiveness to cellular degradation signals can also be engineered into MDP hydrogels through manipulation of the peptide primary sequence. Cellular remodeling of the ECM is a common occurrence in healthy tissue, and the remodeling of scaffolding materials into healthy, functional tissue constitutes the ultimate goal in our tissue engineering strategy. Therefore, a successful MDP hydrogel will be responsive and susceptible to cellular cues for material degradation, and we have engineered this property into MDP hydrogels by including the short amino
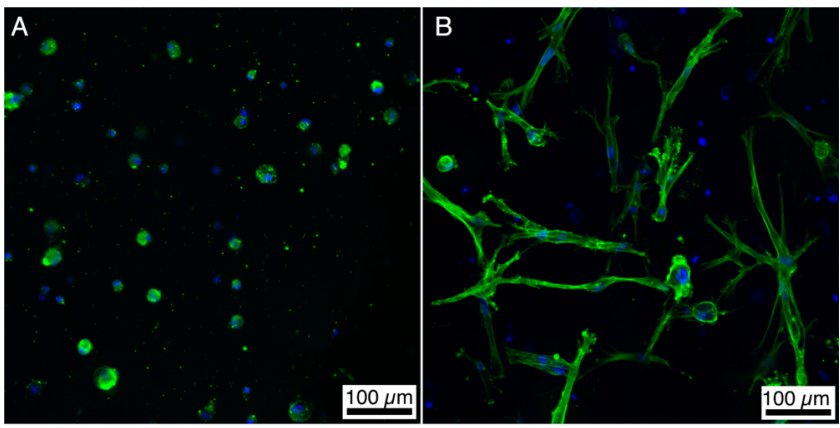

Figure 6. Confocal microscopy images of stem cells from human exfoliated deciduous teeth (SHED) after 11 days of 3D culture in (A) $\mathrm{K}_{2}(\mathrm{TL})_{6} \mathrm{~K}_{2}$ and (B) $\mathrm{K}_{2}(\mathrm{TL})_{6} \mathrm{~K}_{2} \mathrm{GRGDS}$. Cell nuclei are stained with DAPI and appear blue, while the actin cytoskeleton is stained with AlexaFluor 488 phalloidin and appears green. Adapted from ref 13. Copyright 2014 American Chemical Society.

acid sequence LRG. Without major disruption of the material and handling properties, this sequence has been incorporated into the core of the MDP to allow for scission of the peptide bond by the enzyme matrix metalloprotease- $2 .{ }^{25}$ After treatment with MMP-2, cryo-TEM shows that MDP fibers containing the LRG sequence are degraded into amorphous aggregates, while the fibers of peptides without the LRG motif remain intact (Figure $7 \mathrm{~A}, \mathrm{~B}){ }^{16}$ In both in vitro and in vivo environments, cells secrete MMP-2 as they attempt to degrade
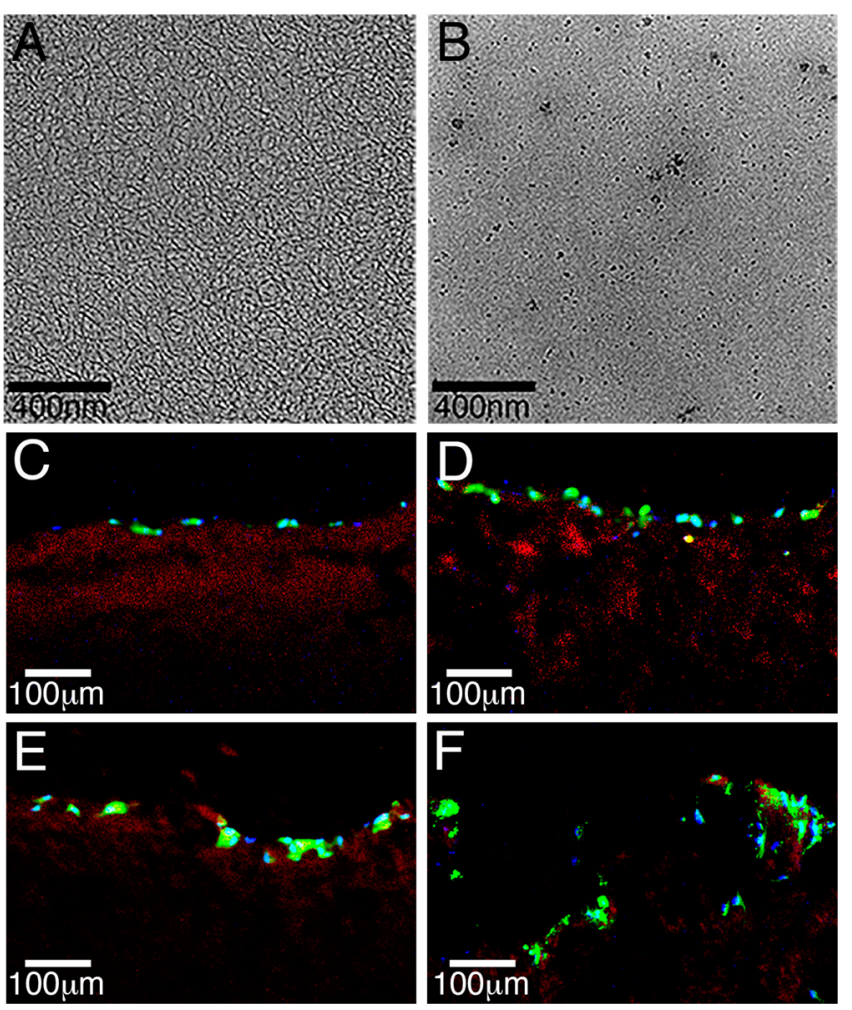

Figure 7. (A, B) Cryo-TEM images of $\mathrm{K}(\mathrm{SL})_{3} \mathrm{RG}(\mathrm{SL})_{3} \mathrm{~K}$ nanofibers showing nanofiber degradation (A) before and (B) after incubation with MMP-2. (C, D) Cell Tracker Green-labeled SHED cells cultured in a monolayer on top of a $\mathrm{K}_{2}(\mathrm{SL})_{6} \mathrm{~K}_{2}$ GRGDS hydrogel after (C) 1 day and (D) 5 days of culture. (E, F) Cell Tracker Green-labeled SHED cells cultured in a monolayer on top of a $\mathrm{K}(\mathrm{SL})_{3} \mathrm{RG}-$ $(\mathrm{SL})_{3}$ KGRGDS hydrogel after (E) 1 day and (F) 5 days of culture. Adapted from ref 16. Copyright 2010 American Chemical Society. 
and remodel the MDP hydrogel, and the responsiveness of the hydrogel to cellular cues stimulating degradation has important consequences for cellular viability and function. We have shown that cells grown in a monolayer on top of the MDP hydrogel are able to migrate into the hydrogel only when the LRG motif is present (Figure $7 \mathrm{C}-\mathrm{F}){ }^{16}$ This has significant implications for tissue regeneration strategies, where infiltration of host cells is imperative for success.

Incorporation of either a cell adhesion motif or an enzymatic cleavage domain serves to create an environment more amenable for cellular functions such as proliferation and matrix remodeling. Essentially, by inclusion of these amino acids the scaffold has been designed at the primary sequence level to respond to the cells' needs and the cues received by cells. In contrast, MDPs can also incorporate bioactive sequences to actively induce a desired cellular process. Through attachment of short bioactive amino acid sequences derived from growth factors, the MDP hydrogel itself can actually bind to and activate cell-surface receptors. While proteins are large and structurally complex molecules, several research groups have sought to identify specific regions of proteins responsible for activation of their target receptors. One specific growth factor mimic of vascular endothelial growth factor (VEGF) has been attached to the C-terminus of the MDP during peptide synthesis. This particular MDP, which we call SLanc, includes both the VEGF mimic to promote angiogenesis and the MMP2 cleavage site to promote scaffold degradation.

Chemical and material characterization of SLanc demonstrates that this mimic can be attached to the MDP without preventing supramolecular assembly, fiber formation, or hydrogelation, suggesting MDP flexibility and tolerance of relatively lengthy mimic sequences. Despite incorporation into the MDP sequence, the VEGF mimic sequence retains its bioactivity, and the SLanc hydrogel itself can activate VEGF receptors to a similar degree as exogenously added growth factor. Subcutaneous injection of the SLanc hydrogel results in mature blood vessels containing an endothelial cell lining, an $\alpha$ SMA muscle layer, and pericytes located within SLanc implants (Figure $8 \mathrm{~A}, \mathrm{~B}) .^{30}$ In a subsequent injury model of hind-limb ischemia, the femoral artery of mice was ligated to prevent blood flow, and SLanc peptide was injected into the quadricep and gastrocnemius muscles $24 \mathrm{~h}$ later. It was shown that this peptide aided in the resolution of hind-limb ischemia, as animals injected with this peptide exhibited a higher perfusion ratio of blood in the ischemic limb and increased treadmill distance running compared with control animals. ${ }^{31}$

We hypothesize that protein-mimicking peptides, such as SLanc, may be able to more robustly drive biological processes than exogenously added growth factor because they present a significantly higher concentration of bioactive signal. Typically, scaffolds used for angiogenesis are loaded with exogenous VEGF at concentrations in the nanomolar range; SLanc, however, presents the bioactive mimic at concentrations in the millimolar range. ${ }^{30}$ Economic feasibility prevents delivery of exogenous growth factor at these concentrations. However, while commercial purchasing of growth factors is rather costly, the cost of including additional amino acids during MDP synthesis is practically negligible. Additionally, as described earlier, growth factors diffuse out of the MDP; in contrast, when the signal is actually contained in the MDP hydrogel itself, the signal remains localized until the material degrades. The success of SLanc suggests that through engineering of the
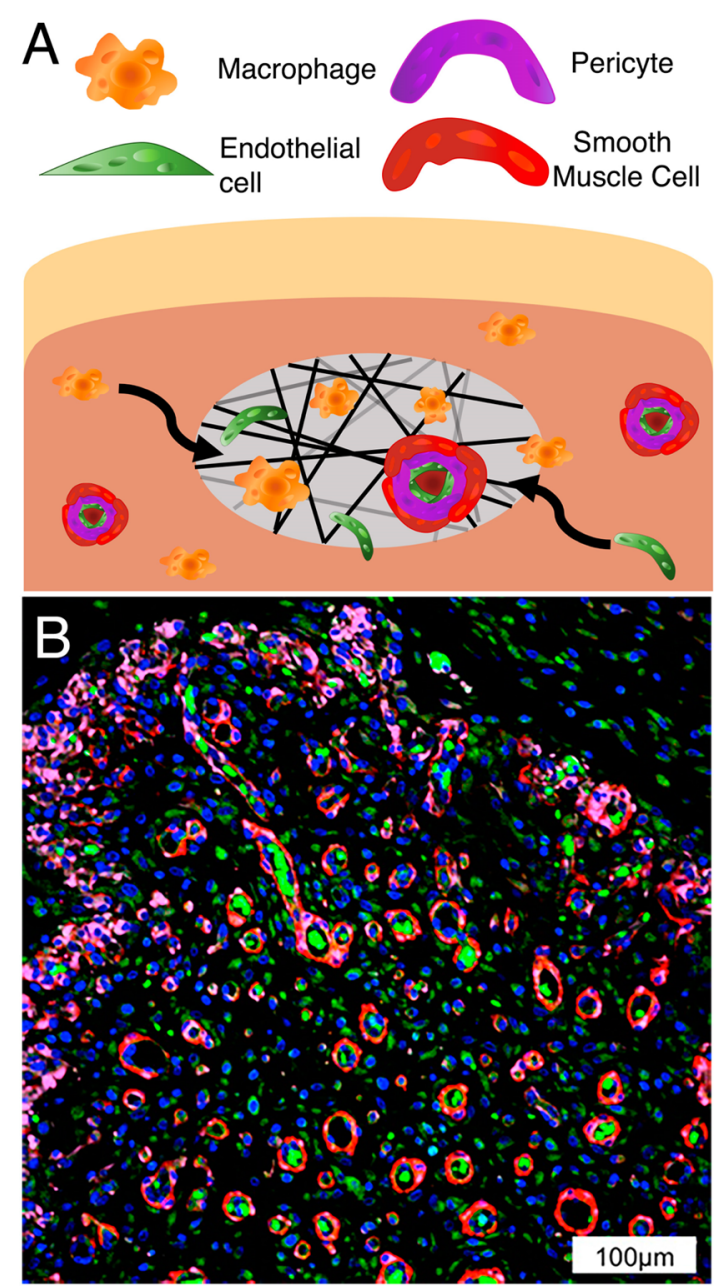

Figure 8. (A) Schematic showing infiltration of cells and blood vessel formation after subcutaneous injection of the SLanc hydrogel. (B) Immunohistochemical analysis of the SLanc implant 7 days after injection. Smooth muscle cells appear red $\left(\alpha \mathrm{SMA}^{+}\right)$, pericyte-like cells appear purple $\left(\mathrm{Nestin}^{+}\right)$, and endothelial cells appear green $\left(\mathrm{CD} 31^{+}\right)$. Adapted from ref 30. Copyright 2015 American Chemical Society.

MDP primary sequence we are able to drive specific cellular processes.

Including biomimics into the MDP primary sequence offers a cost-effective method of driving specific biological responses. We have previously investigated cell adhesion, material degradation, and angiogenesis, but MDPs can be used to drive any process for which a mimic exists. New biological mimic sequences are consistently being identified in the literature, and MDP-mimic hydrogels could be explored for potential use in other applications such as wound healing, resolution of chronic inflammation, and neural development.

The biological studies performed on MDPs have included in vitro, ex vivo, and in vivo analyses. The response to the MDP, molecules delivered by the MDP, or bioactive sequences incorporated into the primary sequence differs drastically depending on the environment. For instance, in vitro studies suggest that incorporation of an enzymatic cleavage motif is necessary for cellular infiltration of the scaffold. ${ }^{16}$ In contrast, hydrogels with or without inclusion of the enzymatic cleavage domain are rapidly populated with cells in in vivo studies. ${ }^{23,28,30}$ Although in vivo cellular infiltration appears to be independent of this motif, the rate of material degradation appears to occur 
much more rapidly when the motif is present. We speculate that the immune and vascular systems are imperative for defining the interaction between cells and our material. This hypothesis is supported through ex vivo experiments, where the MDP appears biocompatible but not highly interactive with native tissue. ${ }^{20}$ While ex vivo studies maintain the complexity of tissue architecture, they lack systemic influences by the immune or vascular systems, suggesting that these systems are key players in defining in vivo results.

\section{CONCLUSION AND FUTURE OUTLOOK}

As a versatile and highly customizable material, MDPs can be tailored to suit a wide range of biological applications. By altering the amino acid composition of the peptide used for hydrogel formation, it is possible to define the chemical, mechanical, and biological properties of the MDP hydrogel. Through the incorporation of multiple bioactive domains, it is possible to create a "smart" scaffold capable of communicating with the biological environment in a complex manner. Ideally, these materials will be able to both respond to cellular signals and influence cell behavior. As biologists gain greater understanding of specific signaling pathways, the molecules involved in these signaling pathways, and the bioactive portions of these molecules, chemists will be able to better design peptide-based materials capable of influencing these pathways. Through an interdisciplinary effort of chemists, biologists, and materials scientists, peptide-based materials have great potential to improve drug delivery and tissue regeneration efforts.

\section{ASSOCIATED CONTENT}

\section{S Supporting Information}

The Supporting Information is available free of charge on the ACS Publications website at DOI: 10.1021/acs.accounts.6b00553.

Tables summarizing all of the published MDP sequences, cell types and cell culture systems, in vivo studies, and molecule release studies (PDF)

\section{AUTHOR INFORMATION}

\section{Corresponding Author}

*E-mail: jdh@rice.edu.

ORCID ${ }^{\circledR}$

Jeffrey D. Hartgerink: 0000-0002-3186-5395

Notes

The authors declare the following competing financial interest(s): J.D.H. has stock options in NangioTx, Inc., which aims to translate some of the technologies presented in this Account toward clinical trials.

\section{Biographies}

Amanda N. Moore received her B.S. in biochemistry from Arizona State University in 2012. She received a master's degree in chemistry from Rice University in 2014 and is currently a Ph.D. candidate at Rice University. Her research interests include the development of selfassembling materials and exploring the biological applications of these materials.

Jeffrey D. Hartgerink is currently a full professor in the departments of chemistry and bioengineering at Rice University, and his research interests lie at the interface of chemistry, biology, and materials science. He completed a dual major in chemistry and biology at
Washington University in St. Louis. He then joined the new interdisciplinary program in chemistry and biology at The Scripps Research Institute, where he earned his Ph.D. under Prof. Ghadiri. Next, he went to Northwestern University, where he completed a postdoctoral fellowship with Prof. Stupp, which culminated with the first publication on self-assembling peptide amphiphiles.

\section{ACKNOWLEDGMENTS}

Work described in this Account was supported in part through three grants: Welch Foundation Grant C1557, NSF CAREER Award DMR-0645474, and NIH Grant R01 DE021798.

\section{REFERENCES}

(1) Koutsopoulos, S. Self-assembling peptide nanofiber hydrogels in tissue engineering and regenerative medicine: Progress, design guidelines, and applications. J. Biomed. Mater. Res., Part A 2016, 104, $1002-1016$.

(2) Ahadian, S.; Sadeghian, R. B.; Salehi, S.; Ostrovidov, S.; Bae, H.; Ramalingam, M.; Khademhosseini, A. Bioconjugated Hydrogels for Tissue Engineering and Regenerative Medicine. Bioconjugate Chem. 2015, 26, 1984-2001.

(3) Webber, M. J.; Berns, E. J.; Stupp, S. I. Supramolecular Nanofibers of Peptide Amphiphiles for Medicine. Isr. J. Chem. 2013, $53,530-554$.

(4) Yokoi, H.; Kinoshita, T.; Zhang, S. Dynamic reassembly of peptide RADA16 nanofiber scaffold. Proc. Natl. Acad. Sci. U. S. A. 2005, 102, 8414-8419.

(5) Schneider, J. P.; Pochan, D. J.; Ozbas, B.; Rajagopal, K.; Pakstis, L.; Kretsinger, J. Responsive Hydrogels from Intramolecular Folding and Self-Assembly of a Designed Peptide. J. Am. Chem. Soc. 2002, 124, 15030-15037.

(6) Rad-Malekshahi, M.; Lempsink, L.; Amidi, M.; Hennink, W. E.; Mastrobattista, E. Biomedical Applications of Self-Assembling Peptides. Bioconjugate Chem. 2016, 27, 3-18.

(7) Matson, J. B.; Stupp, S. I. Self-assembling peptide scaffolds for regenerative medicine. Chem. Commun. 2012, 48, 26-33.

(8) Rubert Perez, C. M.; Stephanopoulos, N.; Sur, S.; Lee, S. S.; Newcomb, C.; Stupp, S. I. The Powerful Functions of Peptide-Based Bioactive Matrices for Regenerative Medicine. Ann. Biomed. Eng. 2015, 43, 501-514.

(9) Boekhoven, J.; Stupp, S. I. 25th Anniversary Article: Supramolecular Materials for Regenerative Medicine. Adv. Mater. 2014, 26, $1642-1659$

(10) Maude, S.; Ingham, E.; Aggeli, A. Biomimetic self-assembling peptides as scaffolds for soft tissue engineering. Nanomedicine 2013, 8 , 823-847.

(11) Dong, H.; Paramonov, S. E.; Aulisa, L.; Bakota, E. L.; Hartgerink, J. D. Self-Assembly of Multidomain Peptides: Balancing Molecular Frustration Controls Conformation and Nanostructure. J. Am. Chem. Soc. 2007, 129, 12468-12472.

(12) Aulisa, L.; Dong, H.; Hartgerink, J. D. Self-Assembly of Multidomain Peptides: Sequence Variation Allows Control over Cross-Linking and Viscoelasticity. Biomacromolecules 2009, 10, 2694-2698.

(13) Kang, M. K.; Colombo, J. S.; D’Souza, R. N.; Hartgerink, J. D. Sequence Effects of Self-Assembling MultiDomain Peptide Hydrogels on Encapsulated SHED Cells. Biomacromolecules 2014, 15, 20042011.

(14) Bakota, E. L.; Sensoy, O.; Ozgur, B.; Sayar, M.; Hartgerink, J. D. Self-Assembling Multidomain Peptide Fibers with Aromatic Cores. Biomacromolecules 2013, 14, 1370-1378.

(15) Colombo, J. S.; Moore, A. N.; Hartgerink, J. D.; D’Souza, R. N. Scaffolds to Control Inflammation and Facilitate Dental Pulp Regeneration. J. Endodontics 2014, 40, S6-S12.

(16) Galler, K. M.; Aulisa, L.; Regan, K. R.; D’Souza, R. N.; Hartgerink, J. D. Self-Assembling Multidomain Peptide Hydrogels: 
Designed Susceptibility to Enzymatic Cleavage Allows Enhanced Cell Migration and Spreading. J. Am. Chem. Soc. 2010, 132, 3217-3223.

(17) Bakota, E. L.; Wang, Y.; Danesh, F. R.; Hartgerink, J. D. Injectable Multidomain Peptide Nanofiber Hydrogel as Delivery Agent for Stem Cell Secretome. Biomacromolecules 2011, 12, 1651-1657.

(18) Ozgur, B.; Sayar, M. Assembly of Triblock Amphiphilic Peptides into One-Dimensional Aggregates and Network Formation. J. Phys. Chem. B 2016, 120, 10243-10257.

(19) Kumar, V. A.; Shi, S.; Wang, B. K.; Li, I.-C.; Jalan, A. A.; Sarkar, B.; Wickremasinghe, N. C.; Hartgerink, J. D. Drug-Triggered and Cross-Linked Self-Assembling Nanofibrous Hydrogels. J. Am. Chem. Soc. 2015, 137, 4823-4830.

(20) Moore, A. N.; Colombo, J. S.; Perez, S. C.; Hartgerink, J. D.; D'Souza, R. N. Ex Vivo Modeling of Multidomain Peptide Hydrogels with Intact Dental Pulp. J. Dent. Res. 2015, 94, 1773-1781.

(21) Bakota, E. L.; Aulisa, L.; Galler, K. M.; Hartgerink, J. D. Enzymatic Cross-Linking of a Nanofibrous Peptide Hydrogel. Biomacromolecules 2011, 12, 82-87.

(22) Caliari, S. R.; Burdick, J. A. A practical guide to hydrogels for cell culture. Nat. Methods 2016, 13, 405-414.

(23) Kumar, V. A.; Taylor, N. L.; Shi, S.; Wickremasinghe, N. C.; D'Souza, R. N.; Hartgerink, J. D. Self-assembling multidomain peptides tailor biological responses through biphasic release. Biomaterials 2015, 52, 71-78.

(24) Wickremasinghe, N. C.; Kumar, V. A.; Hartgerink, J. D. TwoStep Self-Assembly of Liposome-Multidomain Peptide Nanofiber Hydrogel for Time-Controlled Release. Biomacromolecules 2014, 15, 3587-3595.

(25) Galler, K. M.; Hartgerink, J. D.; Cavender, A. C.; Schmalz, G.; D'Souza, R. N. A Customized Self-Assembling Peptide Hydrogel for Dental Pulp Tissue Engineering. Tissue Eng., Part A 2012, 18, 176184.

(26) Kumar, V. A.; Wickremasinghe, N. C.; Shi, S.; Hartgerink, J. D. Nanofibrous Snake Venom Hemostat. ACS Biomater. Sci. Eng. 2015, 1, $1300-1305$.

(27) Wang, Y.; Bakota, E. L.; Chang, B. H. J.; Entman, M.; Hartgerink, J. D.; Danesh, F. R. Peptide Nanofibers Preconditioned with Stem Cell Secretome are Renoprotective. J. Am. Soc. Nephrol. 2011, 22, 704-717.

(28) Wickremasinghe, N. C.; Kumar, V. A.; Shi, S.; Hartgerink, J. D. Controlled Angiogenesis in Peptide Nanofiber Composite Hydrogels. ACS Biomater. Sci. Eng. 2015, 1, 845-854.

(29) Li, I.-C.; Moore, A. N.; Hartgerink, J. D. "Missing Tooth" Multidomain Peptide Nanofibers for Delivery of Small Molecule Drugs. Biomacromolecules 2016, 17, 2087-2095.

(30) Kumar, V. A.; Taylor, N. L.; Shi, S.; Wang, B. K.; Jalan, A. A.; Kang, M. K.; Wickremasinghe, N. C.; Hartgerink, J. D. Highly Angiogenic Peptide Nanofibers. ACS Nano 2015, 9, 860-868.

(31) Kumar, V. A.; Liu, Q.; Wickremasinghe, N. C.; Shi, S.; Cornwright, T. T.; Deng, Y.; Azares, A.; Moore, A. N.; Acevedo-Jake, A. M.; Agudo, N. R.; Pan, S.; Woodside, D. G.; Vanderslice, P.; Willerson, J. T.; Dixon, R. A.; Hartgerink, J. D. Treatment of hind limb ischemia using angiogenic peptide nanofibers. Biomaterials 2016, 98, 113-119. 